Revue d'histoire de l'Amérique française

ZES REVUE D.HISTOIRE DE L'AMÉRIQUE FRANÇAISE

\title{
Un seigneur entrepreneur, Barthélémy Joliette, et la fondation du village d'industrie (Joliette), 1822-1850
}

\section{Jean-Claude Robert}

Volume 26, numéro 3, décembre 1972

URI : https://id.erudit.org/iderudit/303193ar

DOI : https://doi.org/10.7202/303193ar

Aller au sommaire du numéro

Éditeur(s)

Institut d'histoire de l'Amérique française

ISSN

0035-2357 (imprimé)

1492-1383 (numérique)

Découvrir la revue

Citer cet article

Robert, J.-C. (1972). Un seigneur entrepreneur, Barthélémy Joliette, et la fondation du village d'industrie (Joliette), 1822-1850. Revue d'histoire de l'Amérique française, 26(3), 375-395. https://doi.org/10.7202/303193ar d'utilisation que vous pouvez consulter en ligne. 


\section{UN SEIGNEUR ENTREPRENEUR, BARTHÉLEMY JOLIETTE, ET LA FONDATION DU VILLAGE D'INDUSTRIE (JOLIETTE) $1822-1850 \%$}

Jean-Claude Robert

L'histoire d'une ville, surtout à ses débuts, se confond avec celle de son fondateur; mais dans le cas de Joliette et du village d'Industrie, l'extraordinaire identification entre l'homme et son village mérite d'être observée de plus près. A un siècle de distance, serions-nous tenté d'avancer, c'est l'histoire de ces "villes fermées", ces "villes de compagnie", qui a marqué et marque encore les franges du Québec habité. Nous retrouvons ici un phénomène courant dans l'histoire des villes nordaméricaines, mais avec des variantes qui ont un sens et une valeur dans le contexte socio-économique du Bas-Canada de la première moitié du dix-neuvième siècle.

$\mathrm{Au}$ premier chef, et nous insistons sur cette variante, Barthélemy Joliette conçoit ses entreprises comme seigneur de Lavaltrie ${ }^{1}$. La seigneurie constitue son capital: il en tire des revenus en espèces et en nature, des billots, du travail gratuit, et il n'hésite pas à l'hypothéquer au besoin. Enfin, sa notoriété repose sur sa qualité de seigneur qu'il met en évidence à toute occasion (contrats notariés, exigence des droits honorifiques à l'église, etc.). Mais le parti qu'il tire de la seigneurie et l'orientation nettement industrialiste de son activité - n'appelle-t-il pas son village "Industrie" 2 - le distinguent clairement de

\footnotetext{
* Ce texte reprend l'essentiel de notre thèse de maîtrise soutenue à l'été 1971: Jean-Claude Robert, L'activité économique de Barthélemy Joliette et la fondation du village d'Industrie (Joliette) 1822-1850. Thèse de maîtrise ès arts (Université de Montréal, 1971), $183 \mathrm{p}$. Nous tenons à remercier la Société historique de Joliette et particulièrement son secrétaire, Mgr Omer Valois, p.d., pour nous avoir ouvert les archives.

1 Légalement, Barthélemy Joliette ne peut prétendre au titre de seigneur: il n'est que l'époux de la "seigneuresse" (comme on écrivait); et encore, sa femme partage la seigneurie et le titre avec un frère et une sœur. Mais dans les faits, il agit comme s'il était seigneur en titre et en droit; dans la seigneurie, on le considère comme tel, et c'est ce qui importe. 2 Le nom exact est variable: avant 1845, date de l'incorporation de la municipalité sous le nom de Saint-Charles-Borromée-du-Village-d'Industrie, on rencontre Industrie, L'Industrie, Industry Mills; une carte de J. Bouchette de 1831 signale même les "Jolliet Mills".
}

[375 ]

RHAF, vol. 26, no 3 (décembre 1972) 
l'image du seigneur telle que véhiculée dans notre historiographie.

Voilà pourquoi nous le qualifions de seigneur entrepreneur, à défaut d'autre appellation. Nous n'avons pas la prétention de proposer une typologie détaillée pour étudier la société bascanadienne d'avant 1850, mais nous croyons qu'un tel individu en constitue un aspect intéressant. D'autre part, cette étude nous permet d'assister aux dernières années du régime seigneurial qui, en fait, est aboli quatre ans après la mort de Joliette.

\section{I}

Barthélemy Joliette naît le 9 septembre $1789^{3}$ du mariage d'Antoine Jolliet ${ }^{4}$, notaire, et de Catherine Faribault. Antoine Jolliet meurt peu de temps après la naissance de son fils (1791). Après s'être remariée, sa mère vient s'établir dans la région de Chambly. En 1804, le jeune Barthélemy entre en cléricature chez son oncle le notaire Joseph-Edouard Faribault du village de L'Assomption et il reçoit sa commission de notaire le 30 octobre 1810. Soulignons au passage l'importance de l'apprentissage chez le notaire Faribault: notaire fort important à L'Assomption ${ }^{5}$, ce dernier contrôle une bonne proportion des moulins de la région, aussi bien à bois qu'à blé, qu'il possède ou loue. De plus, de 1812 à 1822, Faribault agit comme administrateur de la seigneurie de Lavaltrie. Dès lors, on conçoit facilement que Barthélemy Joliette se soit familiarisé avec le commerce du bois et l'administration de la seigneurie.

Joliette s'intéresse très tôt à la politique. Dès 1814, il se présente comme député. Battu, il conteste avec succès l'élection de son rival; l'année suivante, le même scénario se répète, mais cette fois, Joliette ne parvient pas à obtenir l'annulation de l'élection. Finalement élu en 1820, il n'y siégera pas, la Chambre ayant été dissoute six jours après sa convocation. Il ne tentera pas de retourner à la politique active avant 1830 .

3 Pour les dates et la majorité des faits, nous suivons: abbé Joseph Bonin, Biographie de l'hon. Barthélemy Joliette et de M. le grand vicaire A. Manseau (Montréal, Eusèbe Sénécal, 1874). Complété par les courtes mais excellentes notes biographiques contenues dans: Christian Roy, Histoire de L'Assomption (Montréal, La Commission des fêtes du 250e 1967 ), 216. Pour la vie politique nous avons utilisé: A. DeGrandpré, Notes Joliette 1639-1933, manuscrit (Archives de la société historique de Joliette), (ciaprès ASHJ).

4 Il orthographie également son nom Joliette; Barthélemy signe toujours de cette dernière façon.

5 En 1820, ce village joue le rôle de "centre régional". 
En 1813, Barthélemy a épousé, sous le régime de la communauté de biens, Marie-Charlotte de Lanaudière ${ }^{6}$, fille de Suzanne-Antoinette Margane de Lavaltrie, veuve de C.-G. Tarieu Taillant de Lanaudière. L'épouse apportait en dot la propriété $\mathrm{du}$ quart indivis de la seigneurie de Lavaltrie et les droits au prorata, ainsi qu'une partie des droits que possédait la famille Lavaltrie dans le canton de Kildare, situé à l'arrière de la seigneurie.

Barthélemy Joliette nous apparaît done comme un "notable" du village de L'Assomption. Major et adjudant de milice en 1814 (Lavaltrie), premier syndic pour la reconstruction de l'église en 1819 , il a tâté de la politique avec plus ou moins de bonheur lorsque survient l'événement qui va modifier sa vie: SuzanneAntoinette Margane de Lavaltrie, veuve de Lanaudière, meurt le 22 avril 1822 . L'héritage des Lavaltrie est enfin libre. ${ }^{7}$

A l'aîné des enfants et continuateur de la lignée, PierrePaul de Lanaudière (1794-1832), revient la charge de chef de la famille seigneuriale avec l'héritage de 50\% indivis des biens. Pierre-Georges Roy écrit de lui: "De santé très délicate, monsieur de Lanaudière ne s'occupa jamais d'affaires publiques. Il se contenta de faire valoir sa part de seigneurie." 8 Fait-il vraiment valoir sa part? Nous en doutons. Jusqu'en 1822, le notaire J.-E. Faribault de L'Assomption gérait la seigneurie et, après le décès de la veuve de Lanaudière, Barthélemy Joliette, en plein accord avec les héritiers, devient administrateur fondé de pouvoir. ${ }^{9}$ Bien sûr, la signature de l'aîné apparaît au bas de tous les actes notariés ${ }^{10}$, mais rien ne prouve sa participation aux décisions, comme en témoigne le curé de la paroisse de Saint-Paul:

Mais pour achever de faire connoître la seigneurie, j'ajouterai un mot pour M. Lanaudière. Son nom paroît en tête de tous les actes mais les connoît-il en particulier, en jouit-il ? J'ai peine à le croire. Une personne assez connoissante dans leurs

${ }^{6}$ Le patronyme complet s'écrit: Tarieu Taillant de Lanaudière.

7 Nous devons pour expliquer ceci remonter à l'époque du mariage de B. Joliette. La dot du quart de la seigneurie, donnée par la grand'mère Lavaltrie, contenait une clause lui réservant l'usufruit sa vie durant. La grand'mère meurt en 1815 faisant de sa fille sa légataire universelle. Les époux Joliette lui laissent par la suite l'usufruit, mais lui font signer une reconnaissance de dette peu avant sa mort.

8 P.-G. Roy, La famille Tarieu de Lanawdière (Lévis, 1922), 165.

923 novembre 1825. Acte sous seing privé, Livre A, Seigneurie de Lavaltrie, ASHJ, 88-90.

10 Greffe du notaire Jean-Olivier Leblanc, Archives Judiciaires de Joliette (AJJ). 
arrangemens m'a assuré que $M$. Lanaudière était déjà endossé de plus de 40,000\# envers ses deux beaus frères. C'est un malheur que cet homme n'ait pas le talent de conduire ses affaires, car il est doux et humain; mais les chevaux et les tavernes sont les seules choses qu'il connoisse au monde ${ }^{11}$.

Un coup d'œil sur les comptes de la seigneurie de Lavaltrie nous montre Pierre-Paul de Lanaudière consommant toujours d'avance sa part de la recette tandis que les beaux-frères dont il est question - Barthélemy Joliette et Peter-Charles Loedel - cherchent à réinvestir leur part de recette en plus de réussir à imputer à Pierre-Paul une quote-part plus grande des dépenses tout en l'excluant de certains bénéfices. ${ }^{12}$

La première sœur de Pierre-Paul de Lanaudière, MarieCharlotte (1795-1871), a épousé Barthélemy Joliette en 1813. En fait, elle n'est pas héritière, puisque sur les conseils de son mari elle renonce à la succession de sa mère, déclarant se contenter de ce qu'elle a reçu en dot. ${ }^{13}$ Mariée sous le régime de la communauté de biens, elle laisse son mari gérer le tout; elle est totalement ignorante des affaires du ménage au point de déclarer, en 1852, "qu'elle avait toujours été étrangère aux affaires de son mari qui avaient été bien considérables et avaient embrassé un long espace de temps et remontaient à plus de trente années, [et qu'elle] était presque entièrement ignorante des forces de la succession et de ladite communauté..." 14 Ainsi donc, Marie-Charlotte ne s'occupe presque jamais des affaires de la seigneurie. Dans les actes notariés que nous avons compulsés, nous n'avons rencontré son nom que lorsqu'il s'agissait d'une aliénation définitive d'une partie du patrimoine.

La sœur de Marie-Charlotte et dernière héritière, MarieAntoinette de Lanaudière (1805-1879), lui ressemble fort. C'est une femme effacée dont la chronique locale vante la charité. En 1821, elle a épousé le docteur Peter-Charles Loedel (17951879). Ce dernier partage sensiblement la même situation que Barthélemy Joliette: grâce à leur mariage, tous deux se re-

1123 novembre 1825. Curé John Bellenger à Mgr J.-O. Plessis. Cartable Conversion de Saint-Paul, Registre des lettres 1780-1892. Archives de l'évêché de Joliette (AEJ).

12 Livre A, Seigneurie de Lavaltrie, ASHJ.

13 Donataire d'une partie du douaire de sa mère, elle ne pouvait en plus hériter; d'autre part en renonçant à la succession, elle n'avait pas à en supporter les dettes. Voir Yves-F. Zoltvany, "Esquisse de la coutume de Paris", in RHAF, 25, no 3 (décembre 1971): 365-384.

$141^{\mathrm{er}}$ avril 1852. Intervention. Jos. Eno dit Deschamps, demandeur et la Corporation épiscopale catholique romaine, défenderesse. Cour supérieure \# 531, Archives Judiciaires de Montréal (ci-après AJM). 
trouvent chacun à la tête du quart de la seigneurie. Plus que Pierre-Paul de Lanaudière, Loedel se préoccupera de faire valoir sa part. Associé de Joliette dans la plupart des entreprises, il vient résider au village d'Industrie vers 1825 . Tandis que PierrePaul de Lanaudière vit dans le manoir seigneurial, à Lavaltrie, Joliette et Loedel se font ériger chacun un "manoir" 15 au village d'Industrie.

Mais voyons maintenant cet héritage: la seigneurie de Lavaltrie et les premiers rangs du canton de Kildare en constituent l'essentiel, le reliquat comprenant certains droits dans la seigneurie du Petit-Longueuil et dans celle de Saint-Vallier. La seigneurie de Lavaltrie, bornée d'ouest en est par la seigneurie de Saint-Sulpice et par la seigneurie de Lanoraie, touche le Saint-Laurent au sud et rejoint au nord le canton de Kildare ${ }^{16}$ ou, plus précisément, l' "augmentation sud-est de Kildare". ${ }^{17}$

La seigneurie compte 16 rangs de concessions totalisant 694 lots. ${ }^{18}$ En marge de la seigneurie, il faut ajouter les trois premiers rangs du canton de Kildare qui comprennent 109 lots. ${ }^{19}$ Dans la seigneurie, 323 terres sur 694 n'atteignent pas 60 arpents de superficie tandis que, dans le canton, la grande majorité des terres ont cette mesure. Deux paroisses occupent ce territoire: la plus ancienne, Saint-Antoine-de-Lavaltrie, dont les registres s'ouvrent en 1716 , et une plus récente, située à une douzaine de milles du fleuve, la Conversion de Saint-Paul

15 En principe, il ne peut y avoir qu'un manoir par seigneurie, mais B. Joliette et P.-C. Loedel donnent toujours cette appellation à leur demeure, dans les actes notariés.

16 Arpenté en 1802 et érigé le 24 juin 1803.

17 Cette augmentation (1812), résultant d'une erreur d'arpentage, sera divisée en trois "blocs" de terrain qui finiront tous par devenir partie intégrante de la seigneurie de Lavaltrie, la terre étant concédée de toute manière par les seigneurs de Lavaltrie.

18 Livre terrier de la seigneurie de Lavaltrie (1808), ASHJ. Juin 1829. Aveu et dénombrement de la seigneurie de Lavaltrie, Archives du Québec (A.Q.). - J. Bouchette, A Topographical Dictionary of The Province of Lower Canada (London, Longman, 1832).

19 Bien que ne relevant pas de la tenure seigneuriale, notons que ces lots sont concédés par des baux à rente foncière reprenant toutes les clauses des baux de censives. Voici comment les seigneurs de Lavaltrie procédaient pour concéder des terres tenues en "franc et commun soccage" selon les clauses du régime seigneurial: les formulaires des contrats, imprimés à l'avance, contenaient la clause suivante: les seigneurs appelés pour la circonstance "sieurs comparants" "... lui auraient proposé de lui vendre le dit lopin de terre, pour une certaine somme d'argent en franc et commun soccage, ce que le Preneur ci-après nommé a présentement refusé, et, a préféré (comme plus avantageux pour lui) de prendre le dit Terrain aux charges, clauses, réserves et conditions apposées en ces présentes..." (Greffe du notaire J.-O. Leblanc, AJJ.) 


\section{DIVISIONS TERRITORIALES VERS}

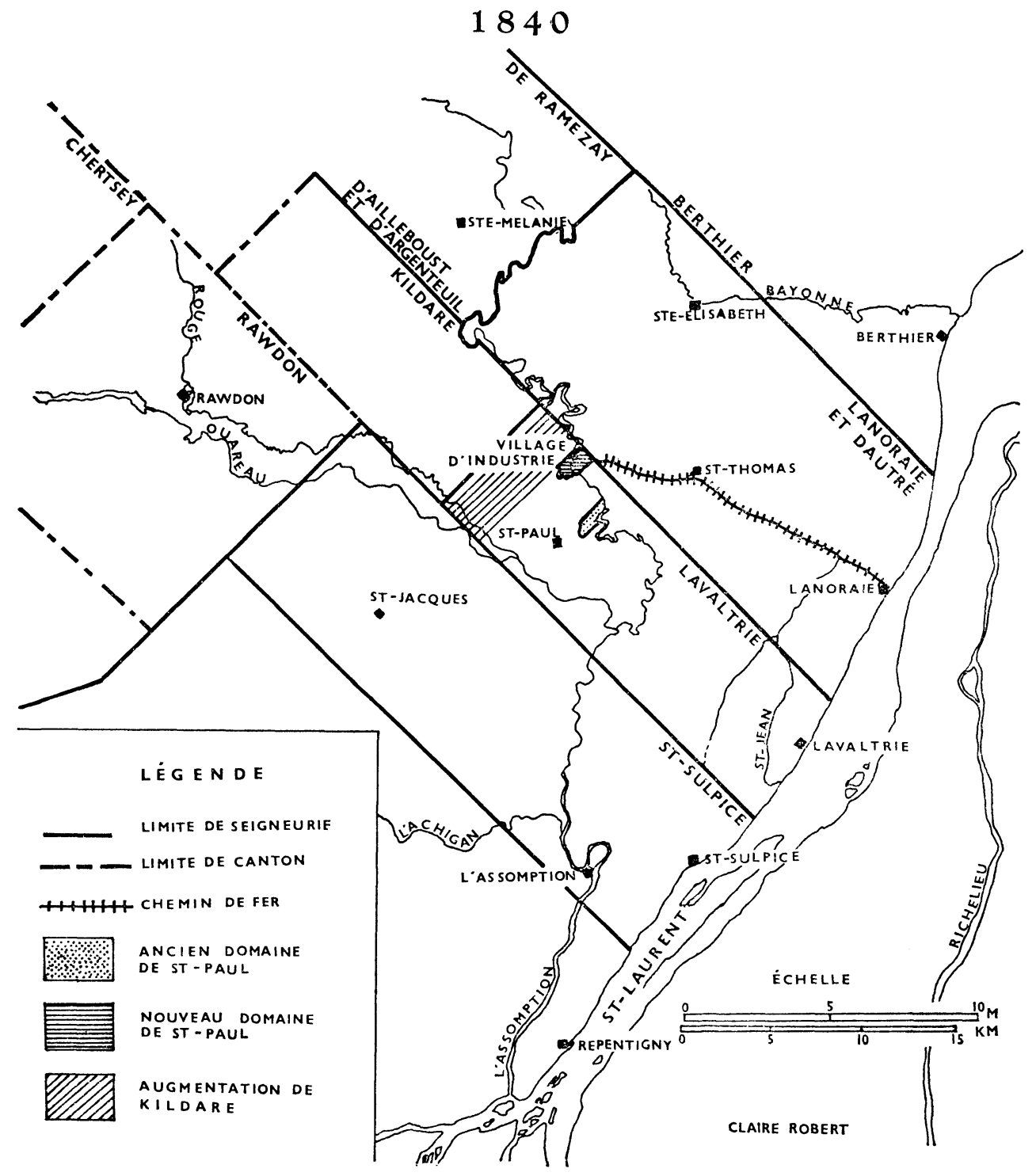

Note: Les frontières seigneuriales sont déduites d'après les lignes actuelles des cantons et d'après la carte de Joseph Bouchette de 1831 (APC, H 11/300-1831). Rappelons qu'il n'existe aucune carte seigneuriale à échelle satisfaisante: trop petites ou trop grandes, elles sont difficilement utilisables. D'autre part, les frontières seigneuriales étaient à ce point floues, que lors de la confection du premier cadastre, il y a un siècle, on n'a même pas retenu leur tracé. 
(appelée également Saint-Paul-d'Industrie, Saint-Paul-de-Lavaltrie), dont les registres s'ouvrent en 1786. Ces deux paroisses correspondent aux deux poussées de colonisation à l'intérieur de la seigneurie: c'est après 1750 que les habitants, venus de la paroisse de L'Assomption, commencent à occuper la seconde. Quant aux trois premiers rangs du canton de Kildare, ils se peupleront entre 1822 et 1825 ; en 1822 , nous ne trouvons que 34 lots concédés sur 109 arpentés.

La seigneurie possède trois moulins. Le premier et le plus ancien, situé sur la petite rivière Saint-Jean, en bordure du fleuve, dessert la paroisse de Saint-Antoine-de-Lavaltrie. Il s'agit d'un moulin à farine et à scie. Le second, un moulin à farine, situé sur le cours de L'Assomption, dans la paroisse de SaintPaul, en dessert les terres. Le troisième, un moulin à scie, cette fois, utilise les eaux du petit ruisseau Saint-Pierre dans la paroisse de Saint-Paul. Il est à noter que ces deux derniers moulins disparaîtront à la suite de la construction des moulins du village d'Industrie. Tous ces moulins ne servent qu'à satisfaire les besoins locaux.

Entre autres constructions importantes, nous ne relevons guère que les églises de Saint-Antoine-de-Lavaltrie et de SaintPaul. A proximité de la première, se trouve le manoir seigneurial. Le village de Saint-Paul, avec ses vingt maisons en $1829^{20}$, est la seule agglomération de la seigneurie. A Lavaltrie, outre l'église et le manoir, se trouvent quatre maisons dont l'une sert de relais pour la poste sur la route Montréal-Québec. ${ }^{21}$

Nous manquons malheureusement de données démographiques complètes; en effet, si nous savons par les réponses des curés à l'enquête de $1820^{22}$ que la paroisse Saint-Paul comptait 2,200 habitants, nous ignorons la population de Saint-Antoine parce que le curé de cette dernière a négligé de la mentionner.

En 1822, la situation financière de la seigneurie montre des arrérages qui nous apparaissent considérables: 370 censitaires doivent la somme de 79,801 livres tournois ou livres anciens cours. ${ }^{23} \mathrm{Il}$ s'agit d'arrérages de cens et rentes et de lods

20 Juin 1829. Aveu et dénombrement de la seigneurie de Lavaltrie, AQ.

$21 \mathrm{~J}$. Samson, Travels in Lower Canada (London, Sir Richard Phillips, 1820), réimpression (Toronto, Coles Publishing Co., 1970), 56. appendice $\mathrm{N}$.

22 Journaux de l'Assemblée législative du Bas-Canada (1821-1822),

23 Seigneurie de Lavaltrie, Livre A, ASHJ, 1-11. Rappelons que le rapport Livre cours actuel ou cours d'Halifax/livre ancien cours ou livre tournoi est de 1 Livre cours actuel $=24$ livres tournois. 
et ventes. Rappelons que depuis dix ans, le notaire J.-E. Faribault, oncle et tuteur de Barthélemy Joliette, administrait la seigneurie; mais nous n'avons pas retrouvé de compte rendu de gestion pour cette période. Par contre, nous savons qu'aucune hypothèque détenue par un étranger à la famille seigneuriale ne grève le passif de la seigneurie de Lavaltrie: les comptes de la succession laissent voir un passif de 40,978 livres tournois dont une hypothèque de 20,093 livres tournois en faveur de Barthélemy Joliette. ${ }^{24}$

Done, en 1822, la seigneurie de Lavaltrie constitue un héritage fort acceptable; elle n'est ni entièrement occupée ni entièrement défrichée, et le bilan financier est positif. Reste à savoir ce qu'en feront les héritiers.

\section{a) $1822-1825$}

Les héritiers de la seigneurie de Lavaltrie, ne se contentent pas de gérer la seigneurie. Ils vont réaliser un projet plus précis et plus rentable: monter une exploitation de bois d'œuvre en utilisant les boisés encore existants sur la seigneurie. Il leur faut donc s'assurer la possession de ces boisés et faire construire un moulin à scie. Dès le début du mois de décembre 1822, leur projet se concrétise.

Par une série d'actes notariés et à titre de coseigneur, administrateur et fondé de pouvoir des seigneurs de Lavaltrie, Barthélemy Joliette fait remembrer un domaine seigneurial dans la paroisse de Saint-Paul. ${ }^{25}$ Ce "nouveau domaine de Saint-Paul", dont une partie deviendra le village d'Industrie, est situé au nord-est de la seigneurie; traversé par la rivière L'Assomption,

24 Il s'agit de l'usufruit dont profite indûment $\mathrm{M}^{\mathrm{me}}$ de Lavaltrie à partir de 1815.

2514 décembre 1822, Cession et abandon par Pierre Desmaroits à P.P.T.T. de Lanaudière, B. Joliette et P.C. Loedel. Greffe du notaire J.E. Faribault, AJJ.

21 décembre 1822, Cession et abandon de François Beaudouin cultivateur à Saint-Paul à P.P.T.T. de Lanaudière, B. Joliette et P.C. Loedel, Greffe du notaire J.E. Faribault, AJJ.

21 décembre 1822, Cession et abandon par Louis Laporte dit St-George à P.P.T.T. de Lanaudière, B. Joliette et P.C. Loedel, Greffe du notaire J.E. Faribault, AJJ.

20 janvier 1823, Echange entre P. de Lanaudière Ec. et autres et François Léonard d. Mondor et uxor, Greffe du notaire J.E. Faribault, AJJ.

Quant à la cinquième terre, nous savons par le livre de mutation, Paroisse de Saint-Paul, ASHJ, qu'elle fut réunie au domaine. 
il offre plusieurs sites propices à l'établissement d'un moulin. L'opération implique cinq terres - toutes concédées mais non défrichées - et ne coûte pas beaucoup aux seigneurs: trois de ces terres leur reviennent en paiement d'arrérages de cens et rentes et une est échangée. Simultanément les seigneurs signent trois contrats pour la construction de la charpente, de la maçonnerie et de l'appareillage mécanique d'un imposant moulin en pierre de trois étages, mesurant 115 pieds sur 50 pieds. ${ }^{26}$ Le coût des trois contrats s'élève à 17,300 livres tournois, sans comprendre le coût des matériaux, et le paiement s'échelonnera sur toute la durée des travaux pour être complété au cours de l'hiver 1824. Les seigneurs ne versent en acompte que 720 livres tournois, ce qui leur permet d'étaler leurs paiements en fonction des autres revenus de la seigneurie. L'édifice abrite un moulin à scie, un moulin à farine à deux moulanges avec toutes ses dépendances, un moulin à clous, un moulin à carder et à fouler, le tout mû par une seule grande roue.

$\mathrm{Au}$ début du mois de juin 1823, l'abattage des arbres commence sur le nouveau domaine de Saint-Paul. De 1823 à 1825, on déboise le domaine; les premiers billots passent par le moulin en mars 1824 et l'ouvrage semble se poursuivre durant tout l'hiver 1825. ${ }^{27}$

Parallèlement à ces activités, Barthélemy Joliette n'oublie pas la gestion de la seigneurie: dès le début, il se préoccupe de toucher les arrérages de cens et rentes et de lods et ventes. En 1823, il entame des procédures contre dix-neuf censitaires. ${ }^{28}$ Il gère si bien ses affaires qu'en novembre 1825, il peut déposer un bilan positif de son administration depuis mai 1822: 128,467 livres tournois de dépenses (comprenant le coût des moulins) contre une recette brute de 140,836 livres tournois. A ce bilan s'ajoute également une somme de 37,563 livres tournois représentant le bois de pin vendu à un marchand de Québec en 1825 . Remarquons la rapidité avec laquelle les arrérages sont payés:

269 décembre 1822. Marché entre François Poitras et P.P. de Lanaudière, B. Joliette et P.C. Loedel Ec. Greffe du notaire J.E. Faribault, AJJ. 13 décembre 1822. Marché entre $D$. Cleveland père et P.P. de Lanaudière, B. Joliette et P.C. Loedel Ecrs. Greffe du notaire J.E. Faribault, AJJ. 23 décembre 1822. Marché entre Antoine Peltier et P.P. de Lanaudière, B. Joliette et P.C. Loedel, Ecrs. Greffe du notaire J.E. Faribault, AJJ. 27 Seigneurie de Lavaltrie. Livre A, ASHJ, 334-335, 350. Les contrats de construction stipulaient que le moulin à scie devait être terminé au printemps 1824 .

28 Repertoire or General Index to all the Causes Instituted in the Superior Term of the Court of King's Bench. In and for the District of Montreal, 1785-1826, AJM. 
39,761 livres tournois dès la fin de 1823 et 27,992 livres tournois, l'année suivante, de sorte qu'au moment du premier bilan de la seigneurie, 74,555 livres tournois d'arrérages sont payées pour un total dû de 79,801 livres tournois. On peut expliquer cette rapidité des rentrées d'argent par le fait que les censitaires peuvent s'acquitter de leurs arrérages en nature (billots) ou en travail, mais surtout par la dureté, voire l'âpreté de Barthélemy Joliette. Lorsqu'en 1825, les seigneurs feront appel à l'évêque pour qu'il recommande au curé de Saint-Paul d'ordonner à ses paroissiens de faire moudre leur blé au moulin banal ${ }^{29}$, le curé de Saint-Paul répondra à son évêque: "Sa gestion de la seigneurie qui ne tendoit rien moins qu'à ruiner ses tenanciers fait bien voir que ce n'est pas la miséricorde ni la compassion qui l'a arrêté. Mais voyant qu'il ne pouvait rien du côté de la loi il a recours à l'autorité de l'Eglise." ${ }^{30}$ Ainsi, la seigneurie de Lavaltrie devient rentable à compter de 1825 .

\section{b) $1826-1832$}

Le nouveau domaine de Saint-Paul étant complètement déboisé à l'automne de 1826, Barthélemy Joliette se tourne vers les seigneuries D'Ailleboust et de Ramezay, toutes deux situées derrière celles de Lanoraie et Dautré qui commencent à peine à se peupler à l'époque. Il obtient de leurs seigneurs, soit des droits de coupe de pin, soit des billots. ${ }^{31}$ Au-delà des seigneuries D'Ailleboust et de Ramezay - comme au-delà du canton de Kildare - s'étendent les "Crown lands" non-arpentées, et à cette époque, un entrepreneur peut couper le bois où il le désire sans craindre l'arrivée intempestive d'un inspecteur du gouvernement. ${ }^{32}$

Après la coupe des arbres, on fait flotter les billots sur la rivière L'Assomption jusqu'aux "moulins d'Industrie"; là, ils sont débités en "deal", c'est-à-dire en madriers de pin de $12^{\prime} \mathrm{x}$ $11^{\prime \prime}$ x 3". Mais ce qu'il faut noter, c'est le trajet suivi par les madriers au départ du moulin: traînés l'hiver par les chemins

29 D'après les textes, il s'agirait d'un meunier peu compétent qui gâchait la farine.

3015 avril 1825. Curé John Bellenger à Mgr Plessis. Cartable Conversion de Saint-Paul-de-Joliette, Registre des lettres 1780-1892, AEJ.

31 Seigneurie de Lavaltrie, Livre A. ASHJ, 297-298. Ce sont là les seules indications sûres que nous possédons; en fait, les chantiers de Joliette, à la recherche de la moindre pinède, remontent graduellement le cours de la rivière L'Assomption.

32 Les archives du ministère des Terres et Forêts, division du Terrier (Québec), regorgent de plaintes de colons s'apercevant que le meilleur bois de leur lot avait été coupé. 
jusqu'au bord de la rivière Ouareau ${ }^{33}$, on les met ensuite sur une barge qui descend vers l'embouchure de la rivière L'Assomption et qui est déchargée au bout de l'île de Repentigny; de là, un second transporteur les prend en charge jusqu'à leur destination finale, à Québec. ${ }^{34}$

En 1826, Barthélemy Joliette manifeste le désir de s'installer à proximité du moulin en faisant entreprendre la construction de deux "manoirs" de pierre, dont l'un est destiné à son beaufrère Peter-Charles Loedel. ${ }^{35}$ Des emplacements sont déjà concédés dans le village d'Industrie et, dès le 11 novembre 1824, une série de sept emplacements trouvent preneur. Ces premiers contrats ne laissent aucun doute sur les intentions de Joliette.

Un emplacement No 13 situé dans le Village d'Industrie, seigneurie de Lavaltrie, de la contenance d'un demi arpent de front sur un arpent de profondeur, borné pardevant à la rue du moulin d'Industrie, par derrière à la rue St Paul, tenant d'un côté au terrain No 10 , d'autre côté au terrain $N^{\circ} 14 .{ }^{36}$

Cet extrait d'un des premiers contrats de concession à l'intérieur $\mathrm{du}$ 'village est fort instructif. Remarquons d'abord que pour la première fois, le nom "village d'Industrie" fait son apparition; en second lieu, notons la présence d'une numérotation de lots ainsi que des noms de rues, ce qui nous permet de conclure à l'existence d'un plan dès 1824 .

Mais avant de poursuivre, voyons un peu la situation du village d'Industrie. Installé sur un des nombreux méandres de la rivière L'Assomption, il ne se trouve éloigné du village de Saint-Paul que d'environ trois milles. La chronique rapporte que des maisons qui se trouvent alors à Saint-Paul sont démolies et remontées au nouveau village d'Industrie. L'“Aveu et dénombrement" de 1829 nous apprend que le village d'Industrie comporte 35 maisons et 29 bâtiments en regard d'une vingtaine

33 Rappelons que la rivière Ouareau se jette dans la rivière L'Assomption et, qu'à la hauteur de Joliette, quelques milles seulement séparent les deux rivières. Quant à la rivière L'Assomption, en aval de Joliette sur quelques milles, elle est parsemée de rapides et son niveau d'eau interdit le passage aux barges même légères.

3423 novembre 1826. Marché entre B. Joliette, P.C. Loedel Ecuiers et J.B. Beaudry père, Frs. Félix Beaudry et Louis Beaudry. Greffe du notaire J.O. Leblanc, AJJ. - 13 février 1827. Marché entre Pierre Talon dit Lespérance et B. Joliette et P.C. Loedel, Ecrs. Greffe du notaire J.O. Leblanc, AJJ. - Il s'agit cette année-là (1827), d'un contrat avec William Price.

3513 janvier 1826. Marché entre B. Joliette, P.C. Loedel et Sr Antoine Peltier, maître maçon. Greffe du notaire J.O. Leblanc, AJJ.

3611 novembre 1824 . Bail à cens par Messrs les Seigneurs de Lavaltrie à J. Bte Gibault. Greffe du notaire J.O. Leblanc, AJJ. 
d'habitations dans le village de Saint-Paul. Ainsi, malgré la proximité d'une agglomération déjà existante qui possède sa propre église, le village d'Industrie fait preuve d'un plus grand dynamisme. Il faut y voir l'effet des moulins d'Industrie qui fixent au nouveau village les employés de Joliette et attirent tous ceux qui sont alléchés par des promesses d'emploi. Barthélemy Joliette cherche également à faire de son village le centre de gravité de sa seigneurie: les cens et rentes doivent se payer au village d'Industrie. Nommé juge de paix en $1826^{37}$, c'est à lui qu'on s'adresse pour régler ses différends. De plus, vers 1825, ayant reçu sa commission de notaire, son ex-clerc JeanOlivier Leblanc, vient s'installer au village d'Industrie. Désormais, il n'est plus nécessaire de parcourir un long trajet jusqu'à L'Assomption ou Berthier pour y passer des actes. Enfin, en 1830, Barthélemy Joliette réussit à se faire élire député du comté. ${ }^{38}$ Cependant, malgré ce succès, demeure une zone d'ombre: c'est en vain qu'il tente d'obtenir la construction d'une église dans son village. Sans doute l'évêque a-t-il été indisposé par une acrimonieuse querelle autour des honneurs dus aux seigneurs dans l'église. ${ }^{39}$ Ajoutons que son puissant voisin, le seigneur Cuthbert, a exercé des pressions sur l'évêque pour faire avorter le projet.40

$\mathrm{Au}$ terme de cette première décennie, un imposant moulin et un village ont surgi là où, dix ans plus tôt, on ne trouvait que cinq terres non défrichées. La seigneurie est devenue rentable comme en témoigne ce bilan général:

$\begin{array}{lrr}\text { Recettes générales }^{41} \text { (en livres tournois) } & \text { recettes } & \text { dépenses } \\ 1^{\text {re }} \begin{array}{l}\text { recette générale }(11 \text { novembre } 1825) \\ \text { mai } 1822 \text { à novembre } 1825\end{array} & 140,836 & 128,467 \\ 2^{\mathrm{e}} \begin{array}{l}\text { recette générale (1 er décembre 1827) } \\ \text { novembre } 1825 \text { à décembre } 1827\end{array} & 58,708 & -\quad{ }^{42}\end{array}$

3726 décembre 1826. Dalhousie. Commission to B. Joliette to take cognizance of small causes in the Seigniory of Lavaltrie. Université de Montréal, Collection Baby, Cote 0, parchemin 2.

38 G. Turcotte, Le Conseil législatif du Québec 1774-1933 (Beauceville, L'Eclaireur, 1933).

39 Joliette et Loedel voulaient bénéficier des honneurs seigneuriaux; en 1826, l'évêque leur répond qu'ils ne peuvent prétendre aux droits honorifiques dus à leurs épouses.

408 juillet 1830 . James Cuthbert à Mgr B.-C. Panet. Cartable Conversion de Saint-Paul de Joliette, registre des lettres 1780-1892, AEJ. Il venait d'ouvrir sa concession du "North Jersey" (Saint-Thomas) et craignait que le village d'Industrie draine ses censitaires.

41 Tiré de: Livre A, seigneurie de Lavaltrie, ASHJ.

42 Il n'y a pas de dépenses au bilan et l'absence des autres livres de compte rend toute vérification impossible. 


\begin{tabular}{|c|c|c|}
\hline $\begin{array}{l}\left.3^{\text {e }} \text { recette générale (20 novembre } 1828\right) \\
\text { décembre } 1827 \text { à novembre } 1828\end{array}$ & 17,421 & 12,659 \\
\hline $\begin{array}{l}\left.4^{e} \text { recette générale ( } 10 \text { décembre } 1829\right) \\
\text { novembre } 1828 \text { à décembre } 1829\end{array}$ & 11,669 & 4,683 \\
\hline $\begin{array}{l}\left.5^{e} \text { recette générale ( } 9 \text { décembre } 1830\right) \\
\text { décembre } 1829 \text { à décembre } 1830\end{array}$ & 17,476 & 4,429 \\
\hline $\begin{array}{l}\text { e recette générale ( } 9 \text { décembre } 1831) \\
\text { décembre } 1830 \text { à décembre } 1831\end{array}$ & 22,085 & 12,382 \\
\hline Total & 268,195 & 162,620 \\
\hline
\end{tabular}

Malheureusement, l'absence de livre de compte du moulin à scie et la pauvreté générale de sources documentaires à ce sujet, ne nous permettent pas de donner des chiffres significatifs quant au commerce du bois: tout au plus pouvons-nous affirmer qu'il est rentable et que les ventes de bois semblent s'échelonner de deux ans en deux ans. ${ }^{43}$

\section{a) l'année 1832}

\section{III}

Cette année marque une étape importante dans la vie de Barthélemy Joliette car, le 10 avril, il est nommé conseiller législatif. Désormais il arborera fièrement son titre d'honorable qui, manifestement, marque la reconnaissance officielle de sa notoriété. Pierre-Paul de Lanaudière meurt le 1er mai, laissant trois enfants mineurs: Barthélemy est nommé tuteur et procureur des enfants. ${ }^{44}$ En rappelant que Pierre-Paul de Lanaudière possédait $50 \%$ de la seigneurie et les droits au prorata, nous voyons que Barthélemy Joliette contrôle maintenant directement $75 \%$ de la seigneurie.

Une des premières mesures prises par Barthélemy Joliette est de procéder à la réfection du terrier de la seigneurie qu'il confie à l'arpenteur Laurent Dorval. ${ }^{45} \mathrm{Au}$ chapitre de la gestion de la seigneurie, une constatation s'impose: les revenus continuent d'augmenter, comme l'indique le seul livre de compte qui nous soit parvenu pour cette seconde période. La moyenne annuelle des revenus bruts pour la première décennie (1822-1832) est de 26,819 livres tournois tandis qu'elle s'élève à 37,643

$43 \mathrm{~A}$ titre d'exemple, pour la période de la première recette générale (mai 1822 à novembre 1825), les cens et rentes en argent rapportent 11,335 livres tournois; les lods et ventes, 8,654 livres tournois; et la vente des madriers du domaine, 37,563 livres tournois.

4420 mars 1851. Compte de tutelle et d'administration rendu par la succession de feu l'hon. B. Joliette. Greffe du notaire Joseph Hilarion Jobin, numéros 7344-7345, AJM.

${ }^{45}$ L. Dorval, Plan de la seigneurie de Lavaltrie, 1833, ASHJ. 
livres tournois pour la période 1832-1844; notons au passage une baisse sensible pour les années 1837 à 1842 .

Si, durant les dix premières années, la seigneurie occupe la première place dans les préoccupations de Barthélemy Joliette, par la suite, son importance tend à diminuer comparativement à l'essor pris par le commerce du bois et les autres affaires. En effet, après 1832, les rentes seigneuriales semblent rentrer régulièrement puisqu'il n'y a pas trace de procès et les quelques "mauvais payeurs" se voient forcés de "vendre" leurs terres aux seigneurs pour éteindre les arrérages qu'ils ont laissé accumuler. D'autre part, le rythme des nouvelles concessions, déjà au ralenti avant 1832, devient de plus en plus irrégulier car les seigneurs n'ont presque plus de terres à concéder.

\section{b) le commerce $d u$ bois}

Ce commerce connaît un tel essor au cours de la période qu'en 1837 on doit installer un second moulin à scie face au premier, sur la rive opposée de la rivière L'Assomption. Mais les sources d'approvisionnement reculent sans cesse vers le haut cours de la rivière de L'Assomption. ${ }^{46}$ Barthélemy Joliette et P.-C. Loedel ${ }^{47}$ auxquels se joint, vers 1837, le marchand Edouard Scallon, doivent recourir aux services de sous-traitants. En 1835, un cultivateur du canton de Kildare s'engage à fournir au moulin 5,000 billots de pin de $12^{\prime} 6^{\prime \prime}$ de longueur et de $22^{\prime \prime}$ de grosseur au petit bout, qu'il tire du canton de Chertsey; dans une série de contrats qu'il donnera peu après, ce cultivateur procédera à une division du travail, se gardant la coupe de 1,400 billots et en répartissant le reste entre sept autres cultivateurs. ${ }^{48}$ Ceci nous permet de saisir sur le vif le mode de vie des cultivateurs de ces régions qui s'occupent de travaux agricoles l'été et deviennent bûcherons l'hiver.

Quant aux chantiers directement exploités par leurs propriétaires, nous en avons retrouvé certaines traces: tantôt sous

${ }_{46}$ D'après l'abbé Joseph Bonin (op. cit., 70), on coupe du bois à l'extrémité du canton de Kildare dès 1832 .

47 Très tôt, le commerce du bois devient distinct de la seigneurie; d'après ce que nous savons, dès que le bois du nouveau domaine de SaintPaul est coupé, soit vers 1825-1826, l'entreprise devient la chose de Joliette et de Loedel. Ils ne s'imputent aucun droit pour l'usage du moulin et vont même dans certains cas jusqu'à faire payer par de Lanaudière une partie des dépenses d'entretien.

4821 octobre 1835. Marché entre A. Marchand, B. Joliette et P.-C. Loedel Ec. Greffe du notaire J.O. Leblanc, AJJ. - 23 décembre 1835. Marché entre A. Marchand et Gabrielle Gagné, J. Charron et Joseph Lemire. Greffe du notaire J.O. Leblanc, AJJ et les quatre contrats suivants. 
forme d'un désaccord entre Barthélemy Joliette et un fournisseur relativement à la livraison des denrées au chantier, tantôt par une série de contrats annuels assurant la livraison des pois ou du foin. Deux "permis de coupe", respectivement de 1842 et de 1845 , nous aident à situer l'aire des chantiers. ${ }^{49}$ Le premier de ces documents autorise l'abattage de 6,000 billots de pin sur des terres de la couronne situées en deçà d'une distance de deux milles de chaque côté de la rivière L'Assomption et de ses affluents, moyennant la somme de $£ 125$ courant ou 3,000 livres tournois; le second "permis" porte la distance à dix milles de chaque côté de la rivière L'Assomption et de ses affluents et ce, sur une longueur de 26 milles en remontant la rivière vers son embouchure. L'approvisionnement ainsi assuré, examinons la capacité des moulins à scie et l'écoulement des madriers sur le marché.

La capacité des moulins s'établit à environ 20,000 billots par année. ${ }^{50}$ De 1839 à 1846 , l'écoulement du bois sur le marché de Québec est assuré par la maison Ryan Brothers qui agit comme courtier. Barthélemy Joliette et ses associés s'engagent à fournir une quantité de madriers évaluée à au moins $£ 2,000$ courant. En retour, Ryan Brothers s'engage à vendre le bois, moyennant une commission de $5 \%$, en plus des frais de quai, tout en avançant à Joliette et à ses associés, une somme de $£ 1,000$ courant. ${ }^{2}$ Pour les deux derniers contrats, Ryan Brothers leur crédite la somme totale de $£ 2,000$ courant, mais hausse sa commission à $71 / 2 \%$. Tout en favorisant l'activité d'un entrepreneur ne possédant pas le capital nécessaire, ce système de crédit ne pouvait, cependant, tolérer une suite de mauvaises années. C'est ainsi qu'en 1841, Joliette et ses associés doivent à Ryan Brothers la somme de $£ 526$ courant; si l'on ajoute cette

4915 janvier 1842. Bond or Obligation of Peter Charles Loedel and of the Hon. B. Joliette, Greffe du notaire J.O. Leblanc, AJJ. -22 avril 1845. Bond or Obligation of the Hon. B. Joliette, P.C. Loedel and Ed. Scallon, Esquires. Greffe du notaire J.O. Leblanc, AJJ.

50 Cette évaluation se fonde sur deux témoignages datés respectivement de 1837 et de 1847. J. Bonin, op. cit., 77.

5218 janvier 1839. Marché Joliette et Loedel et Ryan Brothers. Greffe du notaire Edouard Glackmeyer, numéro 8, AJQ (Archives Judiciaires de Québec).

14 novembre 1839. Marché Joliette et Loedel et Ryan frères. Greffe du notaire Edouard Glackmeyer, numéro 361, AJQ.

26 janvier 1841. Marché Joliette et Loedel et Ryan Brothers. Greffe du notaire Edouard Glackmeyer, numéro 13, AJQ.

27 octobre 1841. Marché Scallon, Joliette et Loedel et Ryan Brothers. Greffe du notaire Edouard Glackmeyer, numéro 271, AJQ.

17 novembre 1842. Marché Scallon, Joliette et Loedel et Ryan Brothers. Greffe du notaire Edouard Glackmeyer, numéro 292, AJQ. 
somme au contrat de l'année, il devient évident qu'il en résulte une accumulation de déficits. ${ }^{53}$

\section{c) les autres projets de Joliette}

Désireux sans doute d'exploiter au maximum le réservoir de main-d'œuvre du village d'Industrie, Barthélemy Joliette fait de nombreux projets et lance de nouvelles entreprises à compter des années 1839-1840. C'est ainsi que, vers 1839, il fait construire une distillerie au village d'Industrie ${ }^{54}$; mais avant même qu'elle soit en opération, il la cède à son associé Edouard Scallon non sans s'être ménagé, par une des clauses contractuelles, le droit de reprendre la moitié de la propriété de la distillerie. ${ }^{55}$ Nous ignorons la raison de cette cession; tout au plus pouvons-nous suggérer, soit un manque de capital liquide, soit la crainte de ruiner ses projets ou l'espoir d'obtenir enfin une église au village d'Industrie. De toute manière, la distillerie disparaît en 1841. Ayant été rasée au sol par un incendie, elle ne sera jamais reconstruite. Il y a cependant apparence qu'elle ait fonctionné environ un an et produit du "whisky" ${ }^{56}$. Après cette expérience, Barthélemy Joliette songe sérieusement à construire une manufacture de verre, mais il ne réalisera pas son projet.57

L'entreprise la plus considérable de Barthélemy Joliette est la construction, de 1847 à 1850 , du "Chemin à lisses de l'Industrie et du Saint-Laurent", également appelé "Chemin à rails de l'Industrie et du Saint-Laurent". Le village d'Industrie, situé à une douzaine de milles à l'intérieur des terres, ne possède pas de moyens de transport donnant facilement accès à d'autres régions. Ainsi le commerce du bois vers Québec se fait par le cours sinueux de la rivière L'Assomption qui coule à la fois vers le SaintLaurent et vers Montréal. Cela entraîne une grosse dépense car le transport $d u$ bois se fait sur une distance d'une vingtaine de milles vers Montréal, distance qu'il faut ensuite refaire en sens inverse pour atteindre Québec ! Des différents tracés, celui qui

53 Les affaires avec Ryan Brothers se terminent en 1846; nous avons retrouvé la mention d'un autre marchand de Québec en 1850, il s'agit de A.H.E. Burstal.

54 J. Bonin, op. cit., 117. C.-H. Panneton, Journal, Carton Joliette témoignages, ASHJ.

5521 janvier 1840. Cession par l'hon. B. Joliette à M. Edouard Scallon. Greffe du notaire J.O. Leblanc, AJJ.

$56 \mathrm{E}$. Scallon s'engage à fournir "... a quantity of whisky or other liquor manufactured at the Distillery established at the said village of Industry ..." 14 novembre 1839. Marché Ed. Scallon et Ryan frères. Greffe du notaire E. Glackmeyer, numéro 360, AJQ.

57 J. Bonin, op. cit., 119. 
est retenu joint le village d'Industrie au village de Lanoraie en passant par le village de Saint-Thomas.

Pour le financement du chemin de fer, Barthélemy Joliette, qui apparaît nettement comme le promoteur de cette affaire, choisit de former une compagnie. Le $1^{\text {er }}$ février 1847, B. Joliette, P.-C. Loedel, Edouard Scallon et Gaspard de Lanaudière ${ }^{58}$ signent un avis demandant l'acte d'incorporation pour la construction d'un "... Chemin de rails dans les paroisses de Lavaltrie, St-Paul et St-Charles Borromée, depuis le fleuve St-Laurent jusqu'à la rivière de l'Assomption, en suivant la ligne qui sépare les seigneuries de Lavaltrie et de Lanorraye..." 59 Le 28 juillet 1847, la "Compagnie du chemin à rails du Saint-Laurent et du village d'Industrie" obtient son incorporation. Son capital est fixé à $£ 12,000$ courant, soit 288,000 livres tournois, divisé en actions de $£ 25$ courant, 600 livres tournois, avec possibilité d'augmenter le capital de $£ 4,000$ courant. $^{60}$ Dès la fin de 1847 , le journal L'Echo des Campagnes annonce que les membres du comité provisoire de la compagnie

... ont fait ouvrir dans le comté de Berthier, deux livres de souscription pour recevoir les signatures des personnes qui désireront devenir souscripteurs à la dite entreprise; lesquels livres de souscription sont déposés, savoir: l'un dans l'office de l'Honorable B. Joliette au Village d'Industrie et l'autre dans la maison de D. M. Armstrong, écuyer, M.P.P. au Village de Berthier, qui sont autorisés à recevoir de telles souscriptions. ${ }^{61}$

En fait, les actions de la compagnie sont offertes à "crédit"; le souscripteur a plus de dix-huit mois (du $1^{\text {er }}$ juin 1848 au $1^{\text {er }}$ décembre 1849) pour effectuer les dix versements de "deux livres dix chelins courant" formant le montant total de 25 livres courant.62 Il semble bien qu'une bonne partie des actions ont été achetées par des habitants du village d'Industrie et par des cultivateurs des paroisses avoisinantes. Dans le greffe du notaire J.-O. Leblanc, nous avons relevé un grand nombre d'actes portant sur ces titres, mais le plus souvent il ne s'agit que de la vente ou de l'échange d'une seule action.

58 Fils de Pierre-Paul de Lanaudière, il est né en 1821 et, à compter de la mort de son père (1832) jusqu'à sa majorité, il est sous la tutelle de B. Joliette.

59 L'Echo des Campagnes, numéro 15 (20 février 1847). Des copies de ce document se trouvent dans ASHJ, dossier Joliette - Chemins de fer; ce dossier fut lui-même publié in extenso par la Société historique de Joliette dans le journal L'Action populaire (mars à avril 1932).

60 J.M. Trout and E. Trout, The Railways of Canada (Toronto, The

Monetary Times, 1871) ; réimpression (Toronto, Coles, 1970), 53.

61 L'E'cho des Campagnes (jeudi 2 décembre 1847).

${ }_{62} L$ 'Echo des Campagnes (mai 1848 et 3 mai 1849). 
La première assemblée générale nomme Barthélemy Joliette au poste de président, P.-C. Loedel, au poste de vice-président et, à titre de trésorier, l'agent d'affaires de Joliette, C.-H. Panneton. Les travaux débutent à l'été de 1848 et, un an et demi plus tard, soit le 24 novembre 1849, la compagnie achète son matériel roulant et ses rails de la "Compagnie du chemin à rails de Champlain et de Saint-Laurent". ${ }^{63}$ A l'hiver de 1850, les travaux sont si avancés que l'on rapporte que, dès l'automne de 1849 , des wagons tirés par des chevaux ont circulé sur une section terminée d'environ huit milles. ${ }^{64}$ Enfin, en mai 1850, peu avant la mort de Barthélemy Joliette, le chemin de fer est inauguré. Mais, apparemment, ce dernier investissement ne devient vraiment fructueux que vers les années 1875 . Notons, en terminant, que ce chemin de fer, d'une longueur de 12 milles, n'est destiné à servir que durant la saison de navigation.

\section{d) Le village d'Industrie 1832-1850}

Parallèlement à la croissance des affaires de Joliette, le village connaît lui aussi des transformations. C'est d'abord la construction d'un marché en 1837, puis l'ouverture de chemins locaux et la construction d'un pont sur la rivière L'Assomption. Mais l'événement le plus important est sans contredit la construction d'une église au village d'Industrie; de 1825 à 1830, nous l'avons vu, Barthélemy Joliette la demande en vain. Ce qu'un "seigneur" de trop fraîche date n'a pu obtenir, devient possible à celui qui est honoré du titre de conseiller législatif et membre du Conseil spécial: en 1841, l'évêque permet la construction d'une chapelle au village. Mais Joliette ne se satisfait pas de si peu et, en 1842, il fait entreprendre à ses frais la construction d'une église. A peine celle-ci est-elle terminée qu'il demande et obtient l'érection canonique d'une nouvelle paroisse du nom de Saint-Charles-Borromée-du-village-d'Industrie (1843). Trois ans plus tard, il édifie un collège confié, en 1847, à la communauté des Clercs de Saint-Viateur, nouvellement arrivée de France. Joliette ne lésine pas: la valeur de l'église et du collège se chiffre à environ 150,000 à 200,000 livres tournois, selon notre évaluation, et ceci, sans tenir compte des deux terres qu'ont

6324 novembre 1839. Obligation de l'Honorable B. Joliette, PeterCharles Loedel et Chs. Bmy Gaspard de Lanaudière Ecuiers à la Compagnie du chemin à Rails de Champlain et St-Laurent. Greffe du notaire J.O. Leblanc, numéro 645 , AJJ. - Les signataires hypothèquent la seigneurie de Lavaltrie en partie, de même que les moulins. Parmi le matériel acheté nous remarquons la présence de la locomotive "Dorchester".

64 J. Loye, Notes, dossier Joliette - Chemins de fer, ASHJ. 
reçues le curé et les religieux, en prévision des frais d'entretien. Pour la construction de l'église, les habitants du village et de la future paroisse ont apporté leur modeste contribution en chaux ou en pierres. ${ }^{65} \mathrm{Il}$ existe même un document intitulé: "Liste de ceux qui n'ont rien fourni pour l'Eglise" ${ }^{86}$, ce qui nous montre bien un trait du caractère de Barthélemy Joliette. Le collège lui-même de deux étages et en pierres fut construit entièrement à ses frais.

L'église et le collège lui servent d'investissements destinés à faire grossir le village. D'ailleurs, de 1843 à 1850, il garde la propriété privée de ces deux édifices malgré le désir du curé Antoine Manseau qui aimerait bien que l'évêque de Montréal et les religieux de Saint-Viateur deviennent propriétaires. Barthélemy Joliette se refuse obstinément à faire une telle donation. ${ }^{67}$ Finalement, peu de temps avant sa mort, il y consentira. ${ }^{68}$

Ce qu'il faut souligner ici c'est que, dans son esprit, le village d'Industrie tout entier constitue non seulement une "entreprise", mais une spéculation conçue dans le but d'attirer et de fixer des gens à demeure. Il escompte ainsi de fructueuses rentrées pour la caisse des seigneurs, surtout à cause des lods et ventes qui ne peuvent qu'augmenter en proportion de la valeur des emplacements. Barthélemy Joliette est, de fait, le maître absolu du village. En l'absence de toute législation municipale, du moins avant 1840 , les articles des contrats de concession énumèrent les charges des preneurs, relativement aux rues, clôtures, égouts, etc. Le tout est placé sous la surveillance des seigneurs qui se réservent en plus le droit de reprendre, moyennant compensation évaluée par eux, tout terrain concédé, si les besoins de leur exploitation de bois l'exigent. Il va sans dire que toute construction de moulin est prohibée.

65 Notons qu'il y avait une carrière utilisée sporadiquement par les seigneurs de Lavaltrie; elle était située sur le bord de la rivière, près du village d'Industrie.

66 Pièce encartée, registre E-32, L'église St Charles Borromée, AEJ.

67 Avant 1850, la paroisse n'a pas de fabrique, et le curé Manseau doit subir l'examen minutieux de ses comptes par B. Joliette, et répondre, acrimonieusement, il est vrai, de sa gestion. -8 janvier 1848. Curé A. Manseau à B. Joliette. Cartable S. Charles de Joliette, registre des lettres, AEJ.

684 février 1850. Donation entre vifs par l'hon. B. Joliette et son épouse à la Corporation épiscopale catholique romaine de Montréal. Greffe du notaire J.O. Leblanc, numéro 699, AJJ. - 4 février 1850 . Donation entre vifs par l'hon. B. Joliette et son épouse à la Corporation des Clercs paroissiaux ou Catéchistes de Saint-Viateur. Greffe du notaire J.O. Leblanc, numéro 698, AJJ. 
En fait, les lotissements progressent comme on peut le voir en comparant un plan d'arpenteur remontant à $1832^{69}$ et une carte conservée aux Archives de la Société historique de Joliette et qui date, croyons-nous, de $1845 .^{70} \mathrm{La}$ comparaison et l'analyse de ces deux documents nous permettent de constater que la superficie occupée par le village fait plus que doubler entre ces deux dates. En 1853, 200 lots sont concédés et on y énumère 208 maisons réparties selon un plan en "damier" sur la dizaine de rues qui sillonnent le village. ${ }^{71}$

Malgré les quelques tentatives pour diversifier l'économie du village, le bois domine toujours, ainsi que le montre un recensement effectué en 1842 par Barthélemy Joliette. Nous y constatons que sur 105 chefs de famille recensés, 29 exercent des métiers directement reliés au travail du bois et 29 autres sont qualifiés de "journaliers"; ces derniers forment la main-d'œuvre occasionnelle employée aux moulins et à des opérations de coupe de bois. ${ }^{72}$ La population du village compte 589 personnes en 1842 et nous l'estimons à environ 1,000 âmes en 1850. Cette population n'est évidemment pas très riche et subit toujours durement les fluctuations de la conjoncture. Le curé A. Manseau écrit en 1843: "Je connais déjà un peu mon village. Les deux tiers sont des journaliers, de pauvres charretiers ou des artisans peu employés. Toujours à peu près incapables de payer l'enterrement de leurs enfants." " 73 Six ans plus tard, en pleine crise du commerce du bois, il écrira: "Les pauvres sont ici en abondance et il y en aura toujours plus ici qu'ailleurs."74

Barthélemy Joliette, qui meurt dans son manoir le 21 juin 1850 , n'eut pas la joie de voir mûrir les fruits de sa grande entreprise; mais ses investissements profitèrent si bien que le village d'Industrie connaîtra un développement rapide entre 1850

6928 avril 1832. Laurent Dorval. "Plan du domaine de Saint-Paul" sur lequel se situait le village d'Industrie. AJM, Cartes et plans, numéro 1345.

70 Plan du domaine de Saint-Paul (Laurent Dorval, s.d.) ASHJ.

7113 juin 1853. Partage de la seigneurie de Lavaltrie. Greffe du notaire Denis Emery Papineau, numéro 3123, AJM.

7229 octobre 1842. Recensement des territoires proposés pour former la nouvelle paroisse de Saint-Charles-Borromée du village d'Industrie. Cartable S. Charles de Joliette, registre des lettres, AEJ.

7321 novembre 1843. Curé A. Manseau à Mgr Bourget. Cartable S. Charles de Joliette, registre des lettres, AEJ.

743 mars 1849. Curé A. Manseau à Mgr Bourget. Cartable S. Charles de Joliette, registre des lettres, AEJ. 
et 1863 , date de son incorporation sous le nom de "ville de Joliette".

Nous venons d'esquisser brièvement la carrière ainsi que les nombreuses entreprises et les réalisations de Barthélemy Joliette. La seigneurie de Lavaltrie joue un rôle primordial dans les affaires de Joliette car elle lui apporte le capital dont il a besoin pour faire fructifier ses affaires. Ce capital se présente sous deux formes principales: par les revenus qu'il en tire et surtout par la solide garantie hypothécaire qui lui permet d'obtenir du crédit. Lorsque vient le temps de construire les moulins en 1823, de signer des contrats de bois entre 1841 et 1846, d'acheter du matériel de chemin de fer en 1849, les contrats se terminent toujours par la clause d'hypothèque des biens. Il administre la seigneurie aussi minutieusement que durement parce qu'il la concoit comme un investissement qui doit rapporter. D'ailleurs, il suit de près toutes ses entreprises, visitant ses chantiers, contrôlant leurs dépenses de nourriture. De son "manoir", édifié tout près des moulins, il peut en surveiller toutes les opérations.

Son caractère dominateur le fait craindre de tous, même des coseigneurs. Le curé Manseau écrit en 1848: "Depuis vingt ans il administre la seigneurie de Lavaltrie. Et je sais que l'intention des coseigneurs est d'attaquer son administration après sa mort." Sachant que Barthélemy contrôle tout, le curé en prévient Mgr Bourget en ces termes:

Il me reste à observer que si on voulait discuter surtout ceci par lettre, il faudra les envoyer par occasions sûres et non par la poste, car bientôt le $\mathrm{Mr}$ en question (Barthélemy Joliette) serait informé de ce qui se passe. Il arrive assez souvent que les lettres soient décachetées, on ne sait pas par qui. ${ }^{75}$

Le curé Manseau demeure le seul d'ailleurs à contester la domination de Joliette; il lutte pour obtenir l'indépendance financière de l'église, ennuyé d'avoir à présenter tous ses comptes au bienfaiteur de la paroisse. Il obtient gain de cause en février 1850. Mais Barthélemy Joliette n'aura pas vu longtemps son pouvoir partagé.

7530 janvier 1848. A. Manseau à Mgr Bourget. Cartable S. Charles de Joliette, registre des lettres, AEJ. 